Bilateral deficits were observed on a test of speed of motor coordination. School records were comparable to the post-trauma test scores. Craniotomy with cyst fenestration and cystoperitoneal shunt resulted in minimal change in size of cyst. Psychological testing at 1 year postsurgery showed no change in language function but significant cognitive improvements in verbal learning, memory, visual-perception/constructional skills, and psychomotor speed, with a 27-point increase in Performance IQ to 117. Reliance on anatomical decompression of the arachnoid cyst may underestimate the efficacy of surgical intervention. (Soukup VM, Patterson J, Trier TT, Chen JW. Cognitive improvement despite minimal arachnoid cyst decompression. Brain Dev Dec 1998;20:589-593). (Respond: Dr Vicki M Soukup, Department of Neurology, University of Texas Medical Branch, Galveston, TX 77555).

COMMENT. Surgical intervention in certain cases of left temporal arachnoid cyst can result in improved cognitive function despite only marginal reduction in size of the cyst. In addition to refractory seizures and headaches, cognitive measures should be used as a functional index of surgical intervention and outcome.

Neurosurgeons in general are reluctant to operate on arachnoid cysts, except in cases complicated by mass effect with seizures and headaches. The report of a Temporal Lobe Arachnoid Cyst/ADHD syndrome, described recently in children with coincidental learning and language disabilities, has emphasized the importance of tests for associated attention deficits and learning disorders in children with arachnoid cysts located in the temporal fossa. (Millichap JG. Temporal lobe arachnoid cyst-attention deficit disorder syndrome. Neurology May 1997;48:1435-1439). PET studies have sometimes demonstrated hypometabolism in cortical areas adjacent to a cyst, and cystoperitoneal shunt can result in improved metabolism and language function. A neurobiologic basis for ADHD, suggested by the TLAC/ADHD syndrome, may prove amenable to surgery in cases of arachnoid cyst complicated by symptoms of a mass effect.

\title{
MPH-INDUCED CHANGES IN RIGHT FRONTAL METABOLISM
}

The effects of methylphenidate (MPH), a drug that increases dopamine, on brain glucose metabolism in 20 cocaine abusers was studied using PET scans and measurements of D2 receptors at the Brookhaven National Laboratory, the State University of New York at Stony Brook, and New York University, NY. MPH increased metabolism in the superior cingulate, right thalamus, and cerebellum. $\mathrm{MPH}$-induced changes in the right striatum and orbitofrontal cortex were associated with cocaine craving, and those in the prefrontal cortex were associated with mood. Compulsive drug abuse in addicted persons may be related to activation of the right striatum and orbitofrontal cortex, areas known to be abnormal in compulsive disorders. The predominant correlation of craving with right but not left brain regions warrants further investigation of laterality of drug responses. (Volkow ND, Wang G-J, Fowler JS et al. Association of methylphenidate-induced craving with changes in right striato-orbitofrontal metabolism in cocaine abusers: Implications in addiction. Am I Psychiatry Jan 1999;156:19-26). (Reprints: Dr Nora D Volkow, Medical Department, Brookhaven National Laboratory, BIdg 490, Upton, NY 11973).

COMMENT. In normal subjects, these authors have reported variable cerebral metabolic responses to MPH; some showing increased metabolism, some decreases, and some no change. Metabolic responses were correlated with measures of dopamine D2 receptors. Similarly, in cocaine abusers, metabolic responses were correlated with dopamine receptors; those with higher D2 levels 
had increased metabolism, and those with lower D2 measures had decreased metabolism. MPH, like cocaine, binds to the dopamine transporter, but their effects on metabolism are not identical. The cocaine craving induced by MPH in cocaine abusers is not associated with an MPH addiction. In fact, if substance abuse becomes a problem in adolescence, it is independent of ADHD and does not involve addiction to MPH (Biederman J et al, 1997, in Millichap JG. Attention Deficit Hyperactivity and Iearning Disorders. PNB Publ, 1998;pp129-130)

The right striato-orbitofrontal regions are thought to be involved in the neuroanatomic and biochemical basis for ADHD. A deficiency in brain dopamine and norepinephrine may explain a loss of inhibition and excessive activity levels in children with $\mathrm{ADHD}$. MPH, by increasing dopamine activity in the right frontal lobe, may normalize motor activity while preserving and heightening the degree of alertness.

\section{PRENATAL PCB AND DIOXIN AND COGNITIVE DYSFUNCTION}

The effects of environmental exposure to polychlorinated biphenyls (PCB) and dioxins on cognitive abilities in Dutch children at 42 months of age were assessed at the Sophia Children's Hospital, Rotterdam, The Netherlands. Maternal plasma and breast milk concentrations were compared with scores of cognitive function. Maternal plasma total PCB concentrations were associated with lower cognitive functioning, and the effect was related to the degree of prenatal in utero exposure. Lactational and current childhood exposure to PCBs and dioxins were not related to 42-month cognitive performance. (Patandin S, Lanting CI, Mulder PGH, et al. Effects of environmental exposure to polychlorinated biphenyls and dioxins on cognitive abilities in Dutch children at 42 months of age. LPediatr Jan 1999;134:33-41). (Reprints: Svati Patandin MD, Department of Pediatrics, Division of Neonatology, SP 3435, Sophia Children's Hospital, PO Box 2060, 3000 CB Rotterdam, The Netherlands).

COMMENT. The developing fetal brain is particularly sensitive to environmental toxins. Prenatal in utero exposure to PCBs causes impaired cognitive performance in children, whereas lactational and childhood exposure may not have adverse effects on learning. These results are in agreement with previous Chinese poisoning and Michigan fish exposure studies. Although deficits are often small, the implications of low-level PCB exposure may be compared to that of lead exposure. Furthermore, the effects on intellectual performance are long-lasting, and ADHD is a potential complication. (Progress in Pediatric Neurology III, PNB Publ, 1997;pp226-8).

\section{ANTIEPILEPTIC DRUGS}

\section{TOPIRAMATE AND OTHER AED EFFECTS ON COGNITION}

The acute and steady-state cognitive effects of three new antiepileptic drugs (AED), gabapentin, lamotrigine, and topiramate, were studied in healthy young adults at the University of Alabama Epilepsy Center, Birmingham, AL. Compared with baseline tests of attention and memory, topiramate (TPM) caused statistically significant declines on measures of attention and word fluency at acute doses, whereas gabapentin (GBP) and lamotrigine (LTG) had minimal effects on performance. Only topiramate subjects had persistent neurocognitive impairments when tested after 2 and 4 weeks of drug administration. The TPM group's verbal fluency rate dropped an average of $50 \%$ per subject, and the visual attention task showed a threefold increase in rate of errors. The adverse effects of 